\title{
On the thermal dependence of the generation of Terahertz radiation by photomixing
}

\author{
S Campbell ${ }^{1}, \mathbf{T}$ Ackemann ${ }^{1}, \mathbf{H}$ Fraser $^{1,3}$, and M Missous ${ }^{2}$ \\ ${ }^{1}$ SUPA and Department of Physics, University of Strathclyde, Glasgow G4 \\ 0NG, Scotland, UK \\ ${ }^{2}$ School of Electrical and Electronic Engineering, The University of Manchester, \\ Manchester, M13 9PL, UK \\ ${ }^{3}$ Now at: Astronomy Division, Department of Physical Sciences, CEPSAR, The \\ Open University, Walton Hall, Milton Keynes MK7 6AA, UK \\ E-mail: thorsten.ackemann@strath.ac.uk
}

\begin{abstract}
We study the temperature dependence of the generation of Terahertz $(\mathrm{THz})$ waves by photomixing in GaAs based antennas, grown at low-temperatures. For excitation with $780 \mathrm{~nm}$ light, the generated photocurrent increases by $15 \%$, if the temperature is increased from $18^{\circ} \mathrm{C}$ to $60^{\circ} \mathrm{C}$, whereas the generated $\mathrm{THz}$ power increases by $28 \%$. The results show that the operation of $\mathrm{THz}$ antennas is robust with respect to changes in the ambient temperature and thus support the possibility of robust, potentially mobile devices without the need for a careful temperature control of the antenna structure.
\end{abstract}

Keywords: THz radiation, cw photomixing, temperature dependence

Submitted to: Semicond. Sci. Technol. 


\section{Introduction}

Terahertz $(\mathrm{THz})$ radiation is electro-magnetic radiation in the frequency range of $0.3-3 \mathrm{THz}$, where many materials show spectroscopic fingerprints but packaging and cloths are transparent. Hence there are great efforts to realize systems for applications in medical diagnosis, material inspection, quality control, short-haul wireless communications and security. Scientifically, terahertz radiation is important for spectroscopic investigations and characterization of modern materials and in astronomy as local oscillator for Terahertz telescopes. Two popular methods for $\mathrm{THz}$ generation are based on photonic sources, in which infrared (IR) lasers generate photocurrents in semiconductor materials with a short lifetime (typically lowtemperature (LT) grown GaAs, e.g. [1]). In photoconductive switching, ultra-short pulses from mode-locked lasers are used for excitation (see, e.g., [2] for a review). In photomixing, the $\mathrm{THz}$ wave is generated from the beating of two $\mathrm{cw}$ IR lasers $[3,4,5,6]$. The $\mathrm{THz}$ radiation inherits the coherence properties of the sources and has hence the prospect for being narrow-band and tunable via a tuning of the frequency difference between the IR lasers. This is imperative for applications relying on high spectral purity such as high-resolution spectroscopy (the linewidth of $\mathrm{THz}$ transitions in low density gases without pressure broadening is less than $1 \mathrm{MHz}$ ) and local oscillators in astronomy. In addition, cw THz systems are highly attractive even for low-resolution spectroscopy and sensing, to avoid the large footprint and costs of mode-locked photo-switching systems. Systems based on thermally tuned distributedfeedback (DFB) lasers $[7,8,9]$ are therefore very attractive for low-cost and mobile $\mathrm{THz}$ spectroscopy, sensing and imaging. Outside a research laboratory, temperature robustness is often an issue. First, the optical beams constitute a significant thermal load which will increase the temperature in the active area of the THz emitter $[10,6]$. Indeed, thermal failure occurs if the temperature difference between the active layer and the substrate reaches about $100 \mathrm{~K}$ [10], which can be counteracted by passive heat sinking techniques $[11,12]$ or array technology $[13,14,15]$. Second, the ambient temperature will be necessarily variable and volatile, in particular in mobile and field applications. As the light-matter coupling in semiconductors decreases with increasing temperature due to broadening of the gain or absorption features, the performance of typical photonic devices degrades with temperature. This raises the question of the need for an expensive and power-hungry active temperature stabilization of the $\mathrm{THz}$ antenna.

We will show here that under typical operating conditions for $\mathrm{THz}$ antennas, there is actually a benefit of increasing the temperature. Though an increase of photocurrent with temperature due to band-gap shrinking was noted before in the literature [6], we are not aware of any systematic investigation. We investigated the temperature range up to $60-70^{\circ} \mathrm{C}$ since it might be relevant in a device with a high-density of components. We demonstrate a photocurrent increase of about $15 \%$ between $18^{\circ} \mathrm{C}$ and $60^{\circ} \mathrm{C}$ and an increase of $28 \%$ in the emitted $\mathrm{THz}$ power. This indicates that the complexity and costs of an active temperature stabilization of the photomixing antennas can be avoided. 


\section{Methods}

\subsection{THz antennas}

The $\mathrm{THz}$ emitters used in this work use standard growth and mounting technology as established as state-of-the-art of the field. The wafers were grown at the University of Manchester and are based on typical LT-GaAs as described, e.g., in [1]. LTGaAs layers were grown in a RIBER V90H MBE system under excess arsenic conditions and at temperatures of approximately $250^{\circ} \mathrm{C}$. The reproducibility of the growth temperature and hence the population of excess point defects was carefully controlled through monitoring the peak split between the GaAs substrate and the asdeposited strained LT-GaAs layer in double crystal X-Ray diffraction measurements. This method ensures that exact replica samples are produced each time as the low growth temperature is notoriously difficult to measure. The samples were then exsitu annealed at $650^{\circ} \mathrm{C}$ for 10 minutes to ensure complete precipitation of the excess arsenic atoms in the GaAs lattice and hence achieve equilibrium defect concentrations. Absorption measurements indicate that the layer thickness is about $0.5 \mu \mathrm{m}$ [16].

The wafers are processed into antenna array based on point dipoles with a $5 \mu \mathrm{m}$ gap at a pitch of $30 \mu \mathrm{m}$ with a total active area of about $1 \times 1 \mathrm{~mm}^{2}$. A microlens array ensures that the light from a broad beam is focused into the gaps of the individual emitters. An aplanatic high resistivity Si lens is mounted onto the back of the wafer to couple out the $\mathrm{THz}$ radiation.

\subsection{Experimental setup}

Two grating-controlled external-cavity diode lasers (ECL) are used as the light sources and provide a tuning range of $8 \mathrm{~nm}$ around $780 \mathrm{~nm}$. The output power is about 35 $\mathrm{mW}$ at an injection current of $100 \mathrm{~mA}$. The two beams were coupled into polarizationmaintaining single-mode fibres and combined in a 50/50 fibre coupler. Each output arm of the coupler produced a total of $20 \mathrm{~mW}$ with a $10 \mathrm{~mW}$ contribution from each beam. The output was then amplified using a Newport TA 7613 tapered semiconductor optical amplifier. A linear polarizer is used after the outcoupler to remove unwanted background of spontaneous emission and to obtain a well-defined polarization state. The output peaked at $500 \mathrm{~mW}$ at $2300 \mathrm{~mA}$ injection current. The output of the fibre was collimated to $1.3 \mathrm{~mm}$ (full width half maximum, FWHM). The beam was then modulated with a mechanical $50 \%$ duty cycle mechanical chopper at $1.3 \mathrm{kHz}$. A half-wave plate placed before the optical isolator was used to balance the fibre-coupled power of the two lasers to maximize the beating contrast. The total power of the beam on the sample was controlled with the amplifier current. Due to the microlens array no focusing is required and the beam width was designed to overfill the array to achieve approximately homogenous driving conditions across the array.

The antenna assembly is mounted onto a suitable sized heat sink to allow the heat generated to dissipate. As the emitter under study is a large-area array, the optical power density and hence the temperature increase due to the optical load is low. The temperature was controlled to an accuracy of $0.1^{\circ} \mathrm{C}$ by a thermo-electric cooler (TEC) feedback system using a thermistor as the sensor. Since the thermistor was not embedded in the antenna assembly itself, it is not possibly to give an accurate local absolute temperature at the antenna. However, the temperature increase of the antenna will be very similar to the temperature increase imposed onto the heat sink. We will refer to the heat sink temperature as ambient temperature in the following 


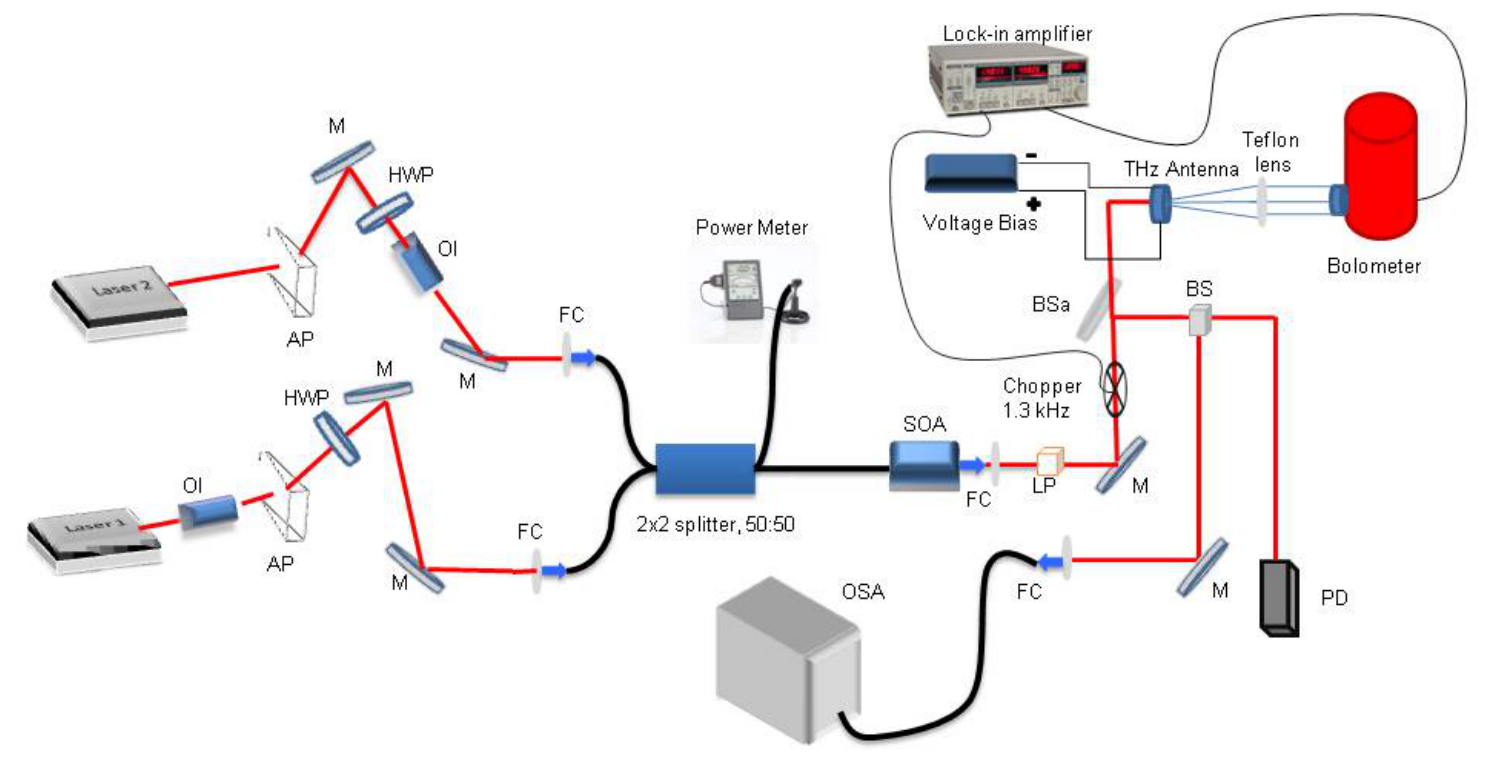

Figure 1. (Colour online) Schematic of experimental setup. M mirror, AP anamorphic prism, OI optical isolator, HWP half-wave plate, L lens, BS 50:50 beam splitter, BSa 90:10 beam sampler, PD photodetector, FC fibre coupling unit, LP linear polarizer. Thick red lines: free space optical beam path, thick black lines: optical fibres, thin black lines: BNC cables, blue: $\mathrm{THz}$ beam.

and use it as a control parameter. The temperature range was between $7^{\circ} \mathrm{C}$ to $67^{\circ} \mathrm{C}$, the limiting factors being the condensation of atmospheric water and thermal damage to the antenna respectively.

The generated $\mathrm{THz}$ radiation is collimated by a silicon lens situated at the back of the antenna which is then focussed using a Teflon lens into the Winston cone of a hot electron InSb bolometer (QMC Instruments Ltd QNI/2), which was used to detect the $\mathrm{THz}$ radiation. The signal was detected with a low-noise pre-amplifier and a lock-in detector. In the following, the values given for the optical input power and the generated photocurrent are averaged over the chopping cycle, i.e. the peak values are a factor of two higher. We will discuss some issues related to the thermal nature of the bolometer signal in the following section.

\subsection{Thermal background}

The bolometric detection method is not able to distinguish between the $\mathrm{THz}$ radiation coherent to the oscillating photocurrent and other thermal signals correlated with the chopper. These are the thermal load coming from the optical beam as well as ohmic heating by the photocurrent. Although the Teflon lens and the filters inside the bolometer (a high-density polyethylene (HDPE) entrance window and the lowtemperature low-pass filters with a $50 \%$ point at $3 \mathrm{THz}$ ) cut out the mid-infrared (MIR) part of the thermal radiation, there is still a thermal tail extending into the $\mathrm{THz}$ region. This can be measured using one laser alone to drive the antenna. This contribution is linear with applied laser power and turns out to generate a lock-in signal of up to $17 \mu \mathrm{V}$ at maximum power $(200 \mathrm{~mW}$ average optical power, $1.35 \mathrm{~mA}$ 
photocurrent) at room temperature. At elevated temperatures $\left(60^{\circ} \mathrm{C}\right)$ it is larger than $30 \mu \mathrm{V}$ (200 mW average optical power, $1.58 \mathrm{~mA}$ photocurrent). The total maximum signal for radiation at $0.5 \mathrm{THz}$ from two-wavelength excitation is about $300 \mu \mathrm{V}$, i.e. the thermal background is small at this frequency. This applies to all the range from microwave frequencies to slightly above $0.5 \mathrm{THz}$, in which the bolometer has a high and approximately constant reponsivity.

However, the responsivity of the bolometer drops off rapidly in the range between 0.5 and $0.7 \mathrm{THz}$. At $1.0 \mathrm{THz}$ it is only about $10 \%$ of its peak value. Hence, the signal coherent with the beat note decreases rapidly at larger frequencies whereas the thermal background stays the same, and a correction of the raw data is necessary. This could be achieved by measuring the thermal background with one laser for every temperature, power and voltage investigated but this was considered not to be worth the considerable effort needed. Instead we choose a simpler method based on a typical responsivity curve of the bolometer. We assume that the bolometer signal, $y$, consists of the thermal background $b$ and the coherent power $s$ multiplied by a sensitivity factor $a, y=b+a s$. If we take $y_{1}, a_{1}$ as the values for $0.5 \mathrm{THz}$ and $y_{2}, a_{2}$ as the values for $1 \mathrm{THz}$ (at the same temperature, optical power and bias voltage), one can obtain the background as

$$
b=\frac{y_{2}-\frac{a_{2}}{a_{1}} y_{1}}{1-\frac{a_{2}}{a_{1}}} .
$$

This can then be subtracted from the raw data. Afterwards, the data for $1 \mathrm{THz}$ can be scaled accounting for the sensitivity drop by multiplying with $a_{1} / a_{2}=1 / 0.107$. We will refer to the raw data as 'lock-in signal' and to the corrected data as ' $\mathrm{THz}$ power (arb. units)' (the numbers given are the bolometer signal in $\mu \mathrm{V}$ expected at the sensitivity for $0.5 \mathrm{THz}$ without the thermal background). As indicated, these considerations are not central to the main findings of this article as the thermal background is negligible at $0.5 \mathrm{THz}$, but needs to be taken into account, if one wants to demonstrate an increase in $\mathrm{THz}$ power also at $1 \mathrm{THz}$ in a semi-quantitative way.

\section{Experimental results}

\subsection{Dark current}

The dependence of the dark current of the antenna array on temperature for a bias voltage of $15 \mathrm{~V}$ is given in Fig. 2. It is $48 \mu \mathrm{A}$ at $17^{\circ} \mathrm{C}$, corresponding to a dark resistance of $0.31 \mathrm{M} \Omega$. Note that this value corresponds to the resistance of all the individual antennas of the array in parallel. It translates to a very good value in the hundreds of $\mathrm{M} \Omega$ range for the single antenna. The dark current increases for increasing temperature as expected for a semiconductor material. In LT-GaAs the main contribution is believed to be the activation of hopping conduction between As precipitates $[17,18]$. Between $17^{\circ} \mathrm{C}$ and $60^{\circ} \mathrm{C}$ the increase is $150 \%$.

\subsection{Dependence on optical input power}

Fig. 3 shows the generated photocurrent in dependence on the incident laser power for different temperatures at a bias of $15 \mathrm{~V}$. The curves are approximately linear at larger photocurrents but show a non-ohmic behaviour at low currents, which is often observed for LT-GaAs antennas. The behaviour is quite similar for a frequency difference of $0.5 \mathrm{THz}$ and $1 \mathrm{THz}$ and the variations between the two cases are indicative 


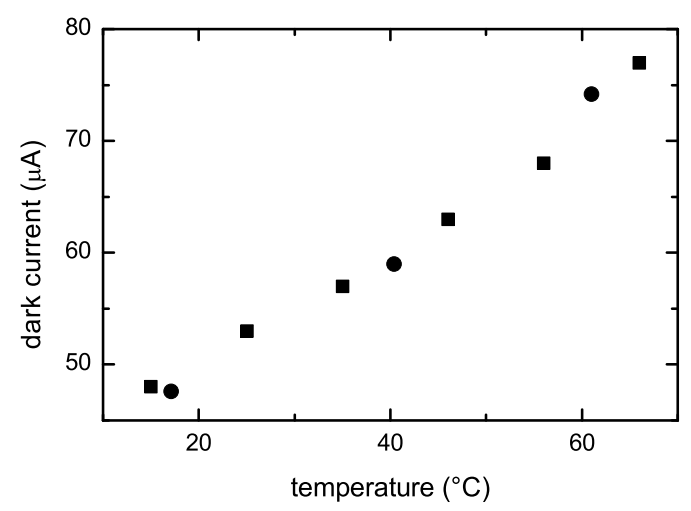

Figure 2. Dark current of antenna array at $15 \mathrm{~V}$ bias. The two different symbols represent measurements taken about 10 months apart.

for the reproducibility of results between different runs and do not represent a genuine difference. There is a significant increase in photo current with increasing temperature. The photocurrent obtained for a frequency separation of $1 \mathrm{THz}$ is very similar. The ratio between the photo current at $60^{\circ} \mathrm{C}$ and $18^{\circ} \mathrm{C}$ is about $15 \%$ at maximum power.

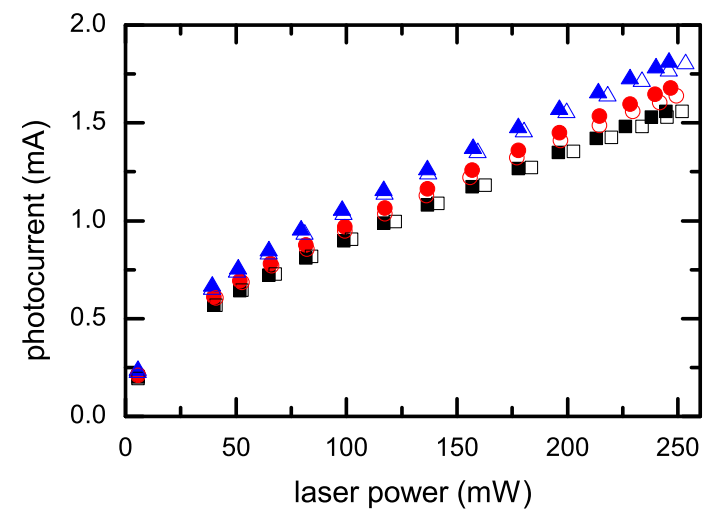

Figure 3. (Colour online) Photocurrent vs. incident laser power for three temperatures at a $15 \mathrm{~V}$ bias (black squares: $18^{\circ} \mathrm{C}$, red circles: $40^{\circ} \mathrm{C}$, blue triangles: $60^{\circ} \mathrm{C}$ ). Solid symbols represent a frequency separation of $0.5 \mathrm{THz}$, open symbols of $1 \mathrm{THz}$. Integration time: $2 \mathrm{~s}$, error bars for the photocurrent noise at constant parameters are negligible.

Fig. 4 shows the generated photocurrent as a function of bias voltage at maximum incident laser power for different temperatures. The curves display a non-ohmic characteristic at low voltage and are reasonably linear at high voltages. Again, there is a consistent and significant increase in photocurrent with temperature. The ratio between the photo current at $60^{\circ} \mathrm{C}$ and $18^{\circ} \mathrm{C}$ is again slightly above $15 \%$ at maximum voltage.

In view of the quite significant increase in photocurrent with temperature, the $\mathrm{THz}$ power emitted was also investigated. Fig. 5 shows the raw (i.e. uncorrected for background) signal of the lock-in detector in dependence on the incident laser power 


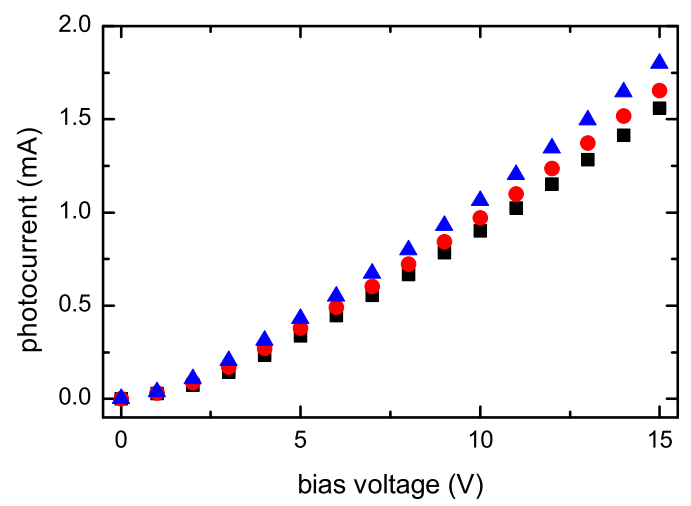

Figure 4. (Colour online) Photocurrent vs. bias voltage at an incident average laser power of $245 \mathrm{~mW}$ for three temperatures (black squares: $18^{\circ} \mathrm{C}$, red circles: $40^{\circ} \mathrm{C}$, blue triangles: $60^{\circ} \mathrm{C}$ ).

for a difference frequency of the IR lasers of $0.5 \mathrm{THz}$ and $1.0 \mathrm{THz}$. In both cases the signal increases superlinearly with power and is higher at higher temperatures for a given power. The absolute signal at $1.0 \mathrm{THz}$ is considerably lower than at $0.5 \mathrm{THz}$, which reflects the reduced sensitivity of the bolometer at this frequency.
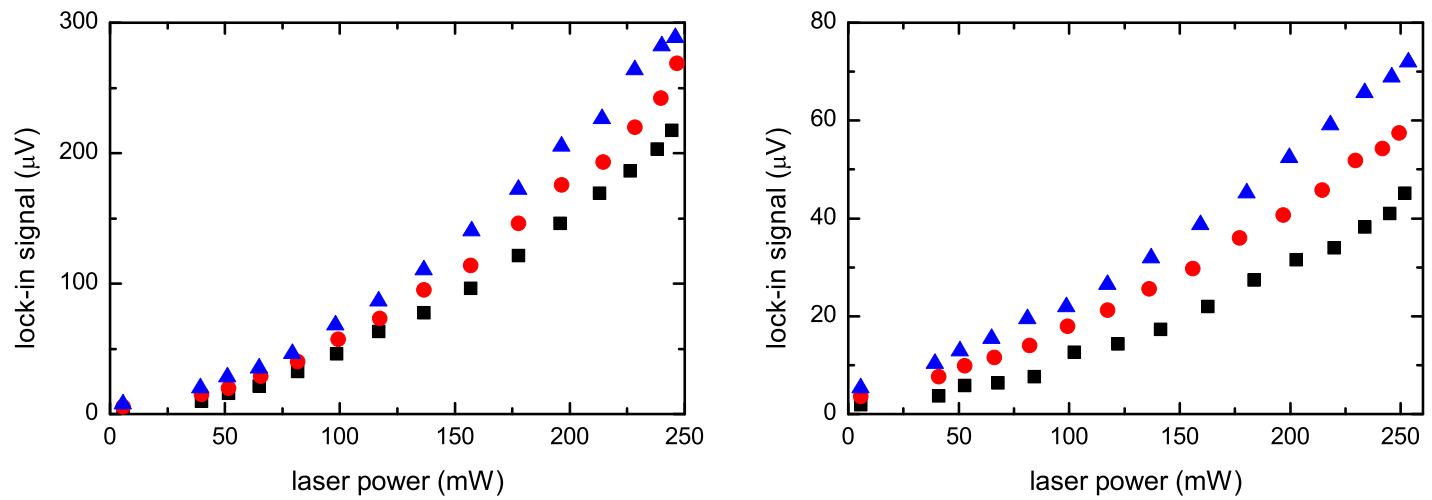

Figure 5. (Colour online) Detected signal after lock-in amplifier vs. incident laser power for three temperatures at a $15 \mathrm{~V}$ bias (black squares: $18^{\circ} \mathrm{C}$, red circles: $40^{\circ} \mathrm{C}$, blue triangles: $60^{\circ} \mathrm{C}$ ). The frequency separation is a) $0.5 \mathrm{THz}, \mathrm{b}$ ) $1 \mathrm{THz}$. Integration time is $1 \mathrm{~s}$ and the readings fluctuate typically about $\pm 2 \mu \mathrm{V}$ (slightly more at high amplitudes) for constant parameters.

Since one expects that the radiated $\mathrm{THz}$ power is proportional to the square of the photocurrent (the field is linear with photocurrent), it makes sense to plot the lock-in signal against photocurrent. The result is shown in Fig. 6. The data for $0.5 \mathrm{THz}$ lie on a single, common characteristic curve independent of temperature, whereas the signal for $1.0 \mathrm{THz}$ increases for increasing temperature, in particular between $18^{\circ} \mathrm{C}$ and $40^{\circ} \mathrm{C}$. Both the 0.5 and $1.0 \mathrm{THz}$ signals increase superlinearly with photocurrent, but the parabolic component is stronger at $0.5 \mathrm{THz}$ than at $1.0 \mathrm{THz}$. These observations indicate that an incoherent background might be important for the $1 \mathrm{THz}$ data. The 
increase in lock-in signal between $18^{\circ}$ and $60^{\circ}$ is about $37 \%$ for $0.5 \mathrm{THz}$ and $59 \%$ for $1 \mathrm{THz}$ for the maximum photocurrent.
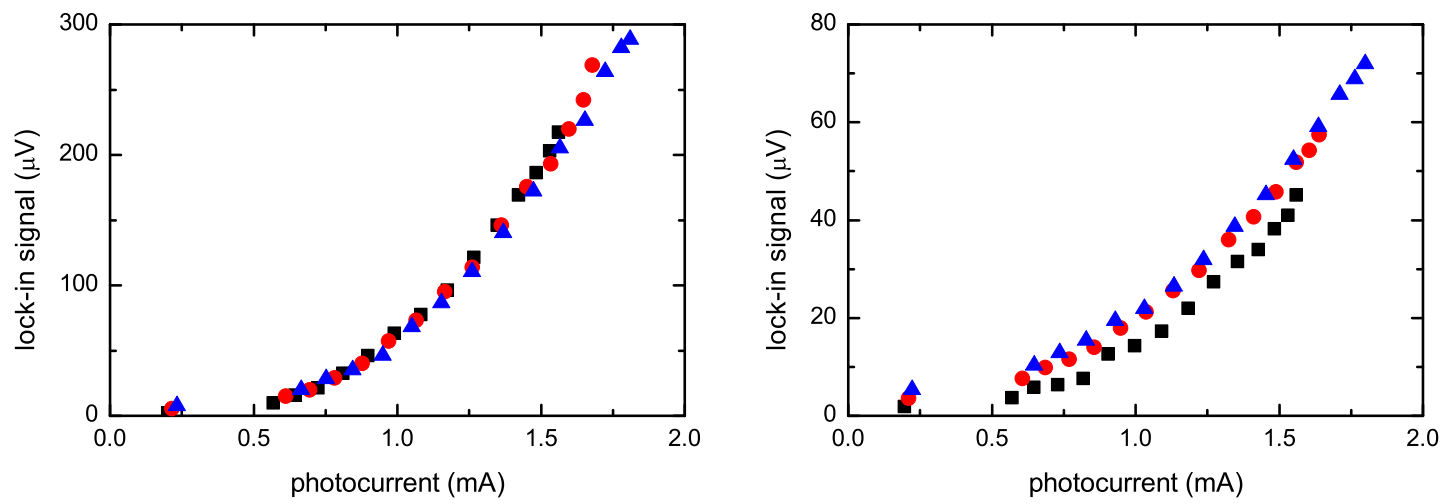

Figure 6. (Colour online) Detected signal after lock-in amplifier vs. photocurrent for three temperatures at a $15 \mathrm{~V}$ bias (black squares: $18^{\circ} \mathrm{C}$, red circles: $40^{\circ} \mathrm{C}$, blue triangles: $60^{\circ} \mathrm{C}$ ). The frequency separation is a) $\left.0.5 \mathrm{THz}, \mathrm{b}\right) 1 \mathrm{THz}$.

Hence we apply the correction procedure explained in Sect. 2.3 and Eq. (1). The offset $b$ obtained increases with laser power and temperature as expected and has also the order of magnitude expected from comparison with the available singlefrequency measurements. The resulting data are presented in Fig. 7. The reduction in amplitude for the data obtained at $0.5 \mathrm{THz}$ (Fig. 7a) is noticable but not large because the bolometer has nearly full sensitivity there. Fig. 7a clearly demonstrates that there is a strong increase of coherent $\mathrm{THz}$ power with photocurrent, and hence with temperature at constant optical power. This increase equates to about $28 \%$ at maximum power, if temperature is raised from $18^{\circ} \mathrm{C}$ to $60^{\circ} \mathrm{C}$. If one divides the increase of the $\mathrm{THz}$ signal by the square of the increase in photocurrent, one obtains values between 0.9 and 1 consistent with the expected quadratic increase of $\mathrm{THz}$ power with photocurrent.

For the data obtained at $1 \mathrm{THz}$, the relative importance of the correction is larger, the peak lock-in signal amplitude drops from $75 \mu \mathrm{V}$ (see Fig. 5b) to about $30 \mu \mathrm{V}$ after subtraction of the offset. We do not present these intermediate data but show in Fig. 7b directly the final result, where also the correction $a_{1} / a_{2}$ for the sensitivity of the bolometer was applied. It is evident that the three characteristics fall on one universal curve. Furthermore, the resulting data have approximately the same amplitude and shape as the data for $0.5 \mathrm{THz}$ in Fig. 7a. There might be a small tendency that the high temperature data $\left(60^{\circ} \mathrm{C}\right)$ are slightly lower than the rest, a tendency already apparent in the uncorrected (see Fig. 5a) as well as the corrected (Fig. 7a) data for $0.5 \mathrm{THz}$. The THz signal increases by $26 \%$ between $60^{\circ} \mathrm{C}$ and $18^{\circ} \mathrm{C}$ at maximum power.

Fig. 8 shows the same data in a double logarithmic plot. The approximately linear dependence indicates polynomial scaling, which motivates fitting to a trial function $P_{\mathrm{THz}}(I)=A\left(I-I_{0}\right)^{s}+b$, where the offsets $I_{0}$ and $b$ are found to be necessary to account for the non-ohmic part of the characteristic (see also [12]). $I_{0}$ is found from a fit of the linear part of the characteristics in Fig. 3 (giving here $I_{0}=0.462 \mathrm{~mA}$, $0.506 \mathrm{~mA}$ and $0.560 \mathrm{~mA}$ for $18^{\circ}, 40^{\circ}$, and $60^{\circ}$, respectively). $s, A$ and $b$ are free 

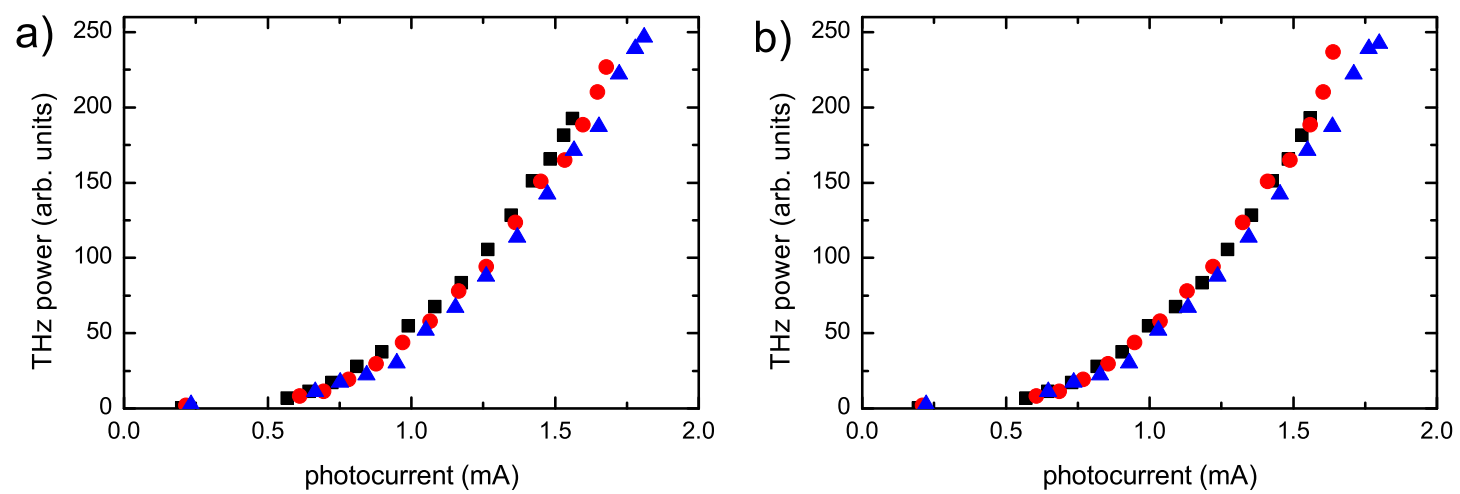

Figure 7. (Colour online) Corrected lock-in signal (signal in $\mu \mathrm{V}$ expected for bolometer sensitivity at $0.5 \mathrm{THz}$ without background, see text for details) proportional to $\mathrm{THz}$ power vs. photocurrent for three temperatures at a $15 \mathrm{~V}$ bias (black squares: $18^{\circ} \mathrm{C}$, red circles: $40^{\circ} \mathrm{C}$, blue triangles: $60^{\circ} \mathrm{C}$ ). The frequency separation is a) $0.5 \mathrm{THz}, \mathrm{b}$ ) $1 \mathrm{THz}$. The figure demonstrates that the $\mathrm{THz}$ power coherent with the beating does not depend on temperature at a fixed photocurrent. The $\mathrm{THz}$ power at maximum optical power (or in general at a given optical power) increases with temperature as the characteristics extend to higher photocurrents.

parameters. The scaling exponent is found to be close to 2 (scattered between 1.8 2.0 for the different temperatures and frequency separations) as expected for quadratic scaling. As an example, the solid lines in Figs. $8 \mathrm{a}$ and $\mathrm{b}$ depict fits of the $40^{\circ}$ data with $s=1.96 \pm 0.08$ for the $0.5 \mathrm{THz}$ data, and $s=1.96 \pm 0.1$ for the $1.0 \mathrm{THz}$ data. This supports that the detected signal corresponds to coherent $\mathrm{THz}$ radiation and indicates that the correction procedure makes sense.
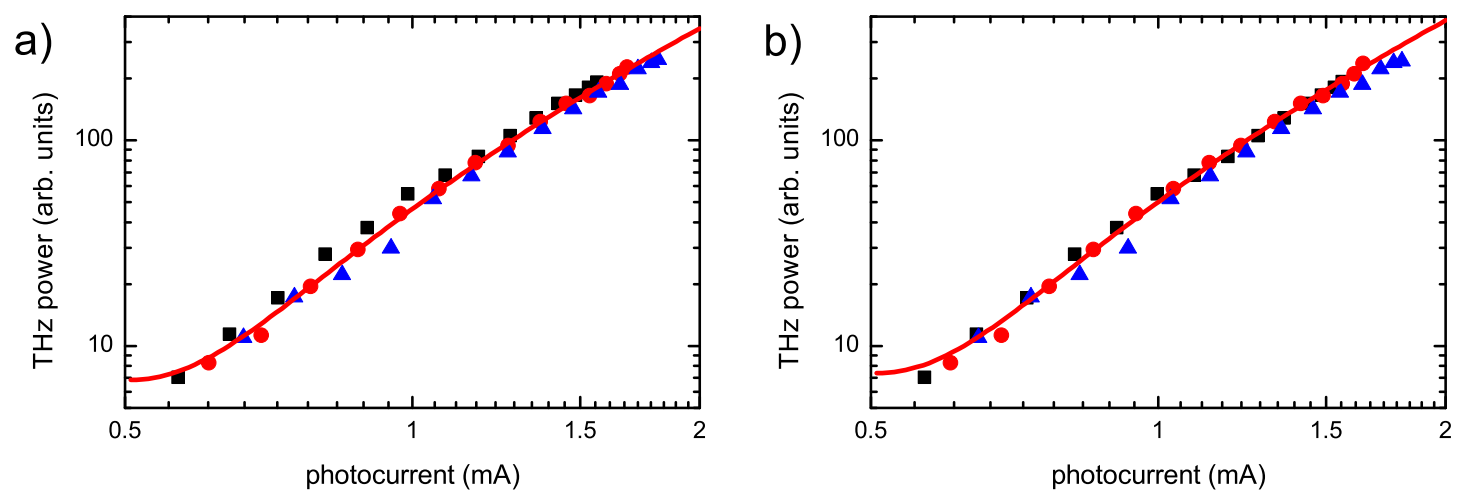

Figure 8. (Colour online) Double logarithmic plot of the corrected (see text) lock-in signal proportional to $\mathrm{THz}$ power (at $0.5 \mathrm{THz}$ ) vs. incident photocurrent for three temperatures at $15 \mathrm{~V}$ bias (black squares: $18^{\circ} \mathrm{C}$, red circles: $40^{\circ} \mathrm{C}$, blue triangles: $60^{\circ} \mathrm{C}$ ). The thick red line is a fit to the data at $40^{\circ} \mathrm{C}$ (details in text). The frequency separation is a) $0.5 \mathrm{THz}, \mathrm{b}) 1 \mathrm{THz}$. 


\subsection{Temperature scan}

The data in the previous subsection established a strong temperature dependence, but only three different temperatures were investigated. Hence we performed a more detailed investigation of the dependence of the $\mathrm{THz}$ output on temperature, fixing the bias voltage at $15 \mathrm{~V}$ and the optical power at the maximal level of $247 \mathrm{~mW}$. Fig. 9a shows the change of photocurrent with temperature for both 0.5 and $1.0 \mathrm{THz}$. It is again apparent that the photocurrent increases with increasing temperature. This increase is approximately linear and about $24-25 \%$ over the range investigated. The variations between the data for the two frequencies are not significant and indicative of the reproducibility of the results regarding the accuracy of temperature control and readout, beam pointing and power fluctuations.

Fig. 9b shows the corresponding increase in $\mathrm{THz}$ power. We are only presenting corrected data. It is superlinear and about $60 \%$ for $0.5 \mathrm{THz}$ and $67 \%$ for $1 \mathrm{THz}$ in the range investigated. Again, in view of the uncertainties of the beam pointing and data treatment the small variations between the data for $0.5 \mathrm{THz}$ and $1.0 \mathrm{THz}$ are not considered to be significant.
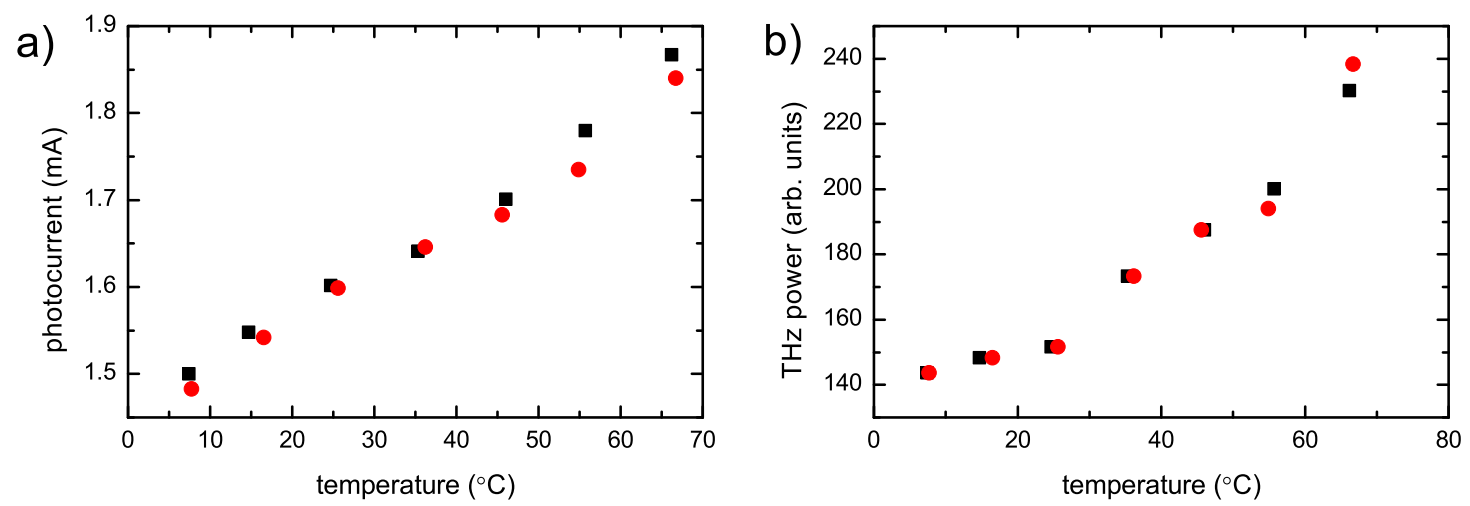

Figure 9. (Colour online) a) Photocurrent vs. temperature and b) corrected lock-in signal proportional to $\mathrm{THz}$ power vs. temperature. Bias voltage $15 \mathrm{~V}$, optical power $247 \mathrm{~mW}$. The squares denote data obtained at $0.5 \mathrm{THz}$ frequency separation, the circles data at $1 \mathrm{THz}$.

In order to support the notion that the detected $\mathrm{THz}$ signal is dominated by the coherent part due to the oscillating photocurrent, it is useful again to plot the $\mathrm{THz}$ power against photocurrent. Fig. 10a shows a plot in linear scale, Fig. 10b in double logarithmic scale. Due to the relatively small changes and large scatter it is difficult to make a quantitative statement but the plot in linear scale shows some curvature in the data, whereas the double logarithmic plot is reasonably straight. The scaling exponents are $s=1.64 \pm 0.1$ for $0.5 \mathrm{THz}$ and $s=1.85 \pm 0.2$ for $1.0 \mathrm{THz}$. It needs to be noted that only a rough (i.e. average) allowance for the non-ohmic parts can be made for these data, since the data are obtained at different temperatures possessing different intercepts $I_{0}$. Overall the data confirm the notion of a significant benefit in $\mathrm{THz}$ power from increasing the ambient temperature. 

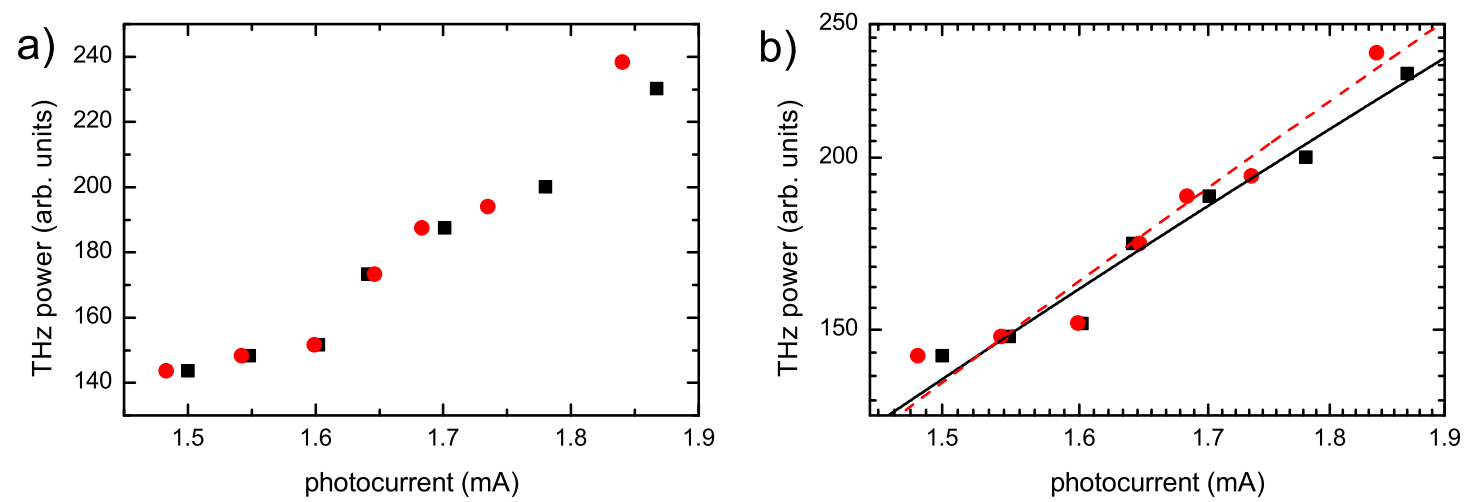

Figure 10. (Colour online) a) Corrected lock-in signal proportional to THz power vs. photocurrent in linear scale and b) in double logarithmic scale. Bias voltage 15 $\mathrm{V}$, optical power $247 \mathrm{~mW}$, scanned temperature range as in Fig. 9. The squares denote data obtained at $0.5 \mathrm{THz}$ frequency separation, the circles data at $1 \mathrm{THz}$. The lines in b) are fits to the curve $y=A\left(x-x_{0}\right)^{s}+b$ with $A, s$ being free fitting parameters as described in the text. $0.5 \mathrm{THz}$ : solid black line, $x_{0}=0.509 \mathrm{~mA}$, $b=6.5 ; 1 \mathrm{THz}$, dashed red line, $x_{0}=0.509 \mathrm{~mA}, b=7.4$.

\section{Discussion}

We had a few antenna arrays at our disposal and all showed an increase of photocurrent between $3 \%$ and $15 \%$ for a temperature increase of $40^{\circ}$. Of these antennas, two showed significant $\mathrm{THz}$ output. Both of these displayed a corresponding increase in $\mathrm{THz}$ output power with the increase in photocurrent. We concentrated in this article on presenting a systematic investigation of one antenna, for the other $\mathrm{THz}$ active antenna the increase in photocurrent is $7 \%$ from $20^{\circ}$ to $60^{\circ}$ and $21 \%$ from $6^{\circ}$ to $80^{\circ}$. The increase in lock-in signal is $15 \%$ from $20^{\circ}$ to $60^{\circ}$ and $46 \%$ from $6^{\circ}$ to $80^{\circ}$ (at $0.68 \mathrm{THz}$ ), in good agreement with the expectation from quadratic scaling.

This increase in $\mathrm{THz}$ generation is directly evident for the data obtained at $0.5 \mathrm{THz}$, but can be demonstrated for data at $1 \mathrm{THz}$ after data correction, accounting for the sensitivity changes in the bolometric detector. This difficulty is only an artefact of the bolometric detection method (in particular at the high frequency), a coherent detection setup, which is the preferred solution for applications anyway, will be much less susceptible to the incoherent background. In coherent detection, the $\mathrm{THz}$ radiation under investigation is mixed with the infrared beams generating it in a second antenna structure (e.g. [5]), thus avoiding the need for cryogenic systems.

The increase in photocurrent independent of the frequency separation indicates that the absorption increases for increasing temperature. One possible mechanism noted in the literature $[10,6]$ is the thermal shift of the bandgap leading to a higher absorption coefficient at a given wavelength. Absorption data for LT-GaAs over a wide energy range were measured in [19]. From these data a value of $17000 / \mathrm{cm}$ for the absorption coefficient of annealed LT-GaAs at $780 \mathrm{~nm}$ is obtained and the the expected absorption is $57 \%$ in a nominally $0.5 \mu \mathrm{m}$ thick layer. The change in absorption is about $70000 / \mathrm{cm} / \mathrm{eV}$ (linear fit to the data in [19]). Taking a common red-shift of the bandgap of GaAs of $0.3 \mathrm{~nm} / \mathrm{K}$, an enhancement of absorption by $4.5 \%$ would be expected from this argument. This is in the lower range what we observe 
experimentally for the photocurrent.

However, the photocurrent does not only depend on the number of generated carriers, but also on the collection efficiency or photoconductive gain (e.g. [20]). Since the electric field in the gap is highly non-uniform and in particular decays with depth, carriers generated close to the surface contribute to the $\mathrm{THz}$ signal more strongly than those generated further down in the material. This is the reason that recessed electrodes provide typically better $\mathrm{THz}$ performance than the standard geometry $[21,22]$. The increased absorption coefficient due to the shift of bandgap will lead to a localization of carrier generation closer to the surface and the resulting increase in collection efficiency might provide the leverage for a superlinear increase in photocurrent with absorption coefficient.

In addition, it seems possible that the enhancement of hopping conductance at elevated temperatures demonstrated for the dark current $[17,18]$ affects also the transport processes under illumination.

\section{Conclusions}

In this paper, we demonstrate a robust increase in photocurrent as well as emitted $\mathrm{THz}$ power for $\mathrm{cw}$ photomixing in LT-GaAs for excitation at $780 \mathrm{~nm}$. Independent of a detailed understanding of the mechanisms, this is an interesting result as it demonstrates that working at moderately elevated temperatures does not provide an obstacle for the operation of $\mathrm{THz}$ emitters. Thus no expensive temperature control is needed facilitating widespread use and potentially mobile, even hand-held devices. Using lower wavelength lasers at $760 \mathrm{~nm}$ should enhance performance as well as enabling a cross-check of the mechanisms involved. Pump-probe measurements of the temperature dependence of the carrier lifetime, preferentially under biased conditions, would be another way to investigate mechanisms involved.

\section{Acknowledgements}

The Strathclyde group gratefully acknowledges support from Royal Society (London) and a SUPA Start knowledge exchange grant. S.C. was partially supported by an EPSRC DTA. We are grateful to J. Roulston, Scimus Solutions Inc., for fruitful discussions.

[1] Gregory I S, Baker C, Tribea W R, Evans M J, Beere H E, Linfield E H, Davies A G and Missous M 2003 Appl. Phys. Lett. 83 4199-4201

[2] Jepsen P U, Cooke D G and Koch M 2011 Laser Photonics Rev. 5 124-166

[3] Brown E R, McIntosh K A, Nichols K B and Dennis C L 1995 Appl. Phys. Lett. 66 285-287

[4] McIntosh K A, Brown E R, Nichols K B, McMahon O B, DiNatale W F and Lyszczarz T M 1995 Appl. Phys. Lett. 67 3844-3846

[5] Gregory I S, Tribe W R, Baker C, Cole B E, Evans M J, Spencer L, Pepper M and Missous M 2005 Appl. Phys. Lett. 86204104

[6] Preu S, Döhler G H, Malzer S, Wang L J and Gossard A C 2011 J. Appl. Phys. 109061301

[7] Deninger A J, Göbel T, Schönherr D, Kinder T, Roggenbuck A, Köberle M, Lison F, MüllerWirts T and Meissner P 2008 Rev. Sci. Instrum. 79044702

[8] Stanze D, Deninger A J, Roggenbuck A, Schindler S, Schlak M and Sartorius B 2011 J Infrared Milli Terahz Waves 32 225-232

[9] Kim N, Han S P, Ko H, Leem Y, Ryu H C, Lee C, Lee D, Jeon M, Noh S and Park K H 2011 Opt. Exp. 19 15397-15403

[10] Verghese S, McIntosh K A and Brown E R 1997 Appl. Phys. Lett. $712743-2745$

[11] Matsuura S and Ito H 2005 Generation of cw Terahertz radiation with photomixing (Springer, Topics in Applied Physics vol 97) (Springer) pp 157-202 
[12] Ackemann T, Alduraibi M, Campbell S, Keatings S, Luke Sam P, Fraser H, Arnold A S, Riis E and Missous M 2012 J. Appl. Phys. 112123109

[13] Dreyhaupt A, Winnerl S, Dekorsy T and Helm M 2005 Appl. Phys. Lett. 86121114

[14] Saeedkia D, Mansour R R and Safavi-Naeini S 2005 IEEE Trans. Ant. Prop. 534044

[15] Matthäus G, Nolte S, Hohmuth R, Voitsch M, Richter W, Pradarutti B, Riehemann S, Notni G and Tünnermann A 2008 Appl. Phys. Lett. 93091110

[16] Roulston J and Missous M 2011 Unpublished.

[17] Look D C, Walters D C, Manasreh M O, Sizelove J R, Stutz C E and Evans K R 1990 Phys. Rev. B 42 3578-3581

[18] Kordoš P, Marso M, Förster A, Darmo J, Betko J and Nimtz G 1997 Appl. Phys. Lett. 71 $1118-1120$

[19] Dankowski S U, Streb D, Ruff M, Kiesel P, Kneissl M, Knüpfer B, Döhler G H, Keil U D, Sorenson C B and Verma A K 1996 Appl. Phys. Lett. 68 37-39

[20] Brown E R 199 Appl. Phys. Lett. 75 769-781

[21] Mikulics M, Wu S, Marso M, Adam R, Förster A, Hart A, Kordoš P, Lüth H and Sobolewski R 2006 IEEE Photon. Tech. Lett. 18 820-822

[22] Kordoš P, Marso M and Mikulics M 2007 Appl. Phys. A 87 563-567 